\title{
Amylase gene duplication: an ancestral trait in the Drosophila melanogaster species subgroup
}

\author{
O. Daïnou* \\ M. L. Cariou* \\ J. R. David* and \\ D. Hickey ${ }^{\dagger}$
}

\author{
* Laboratoire de Biologie et Génétique Evolutives, \\ CNRS, 91190 Gif-sur-Yvette, France. \\ $\uparrow$ Department of Biology, University of Ottawa, \\ Ottawa, Ontario, K1N 6N5, Canada.
}

Electrophoretic polymorphism of amylases was studied in 45 geographic populations of the two cosmopolitan sibling species, $D$. melanogaster and $D$. simulans, and in one to three populations or strains of six other species in the $D$. melanogaster subgroup. Two species, $D$. erecta and $D$. orena, for which only a few strains were available were monomorphic. In the other species 2 or 3 amylase variants were identified while in $D$. melanogaster, 12 electrophoretic variants were characterized. Altogether 17 different amylase isozymes have been observed. The contrast in the level of polymorphism between $D$. melanogaster and the other species cannot be explained simply by the occurrence of a duplication in the former species. Genetic analysis demonstrated the existence of a duplication in at least 4 other species, namely $D$. simulans, $D$. mauritiana, $D$. yakuba, and $D$. teissieri. It is therefore suggested that the duplication occurred in a common ancestor and the phylogenetic implications of these observations are discussed.

\section{INTRODUCTION}

Amylase was one of the first Drosophila enzymes to be studied genetically (Abe, 1958; Kikkawa, 1960). With the introduction of electrophoretic methods, a series of variants were found, apparently corresponding to the expression of different alleles (Kikkawa, 1964; Doane, 1965). Some homozygous flies were found to produce two different proteins, suggesting a duplication of the structural locus. This was demonstrated by Bahn (1967) who observed very rare recombinants between electrophoretic alleles showing a very close linkage between the duplicated loci, (genetic distance less than 0.01 centimorgan). Definitive proof of the duplication was provided by molecular experiments (Levy et al., 1985).

In early studies, different drosophilid species were investigated for evidence of an amylase $(A m y)$ structural gene duplication (summary in Doane, 1969) but no single individual exhibited three different amylases isozymes and inbreeding, when possible, never led to the production of homozygous flies with two different isozymes. It was thus concluded that the $D$. melanogaster case was unique, suggesting the duplication to be a recent, or derived, genetical trait. However, in Doane's survey, only one species closely related to $D$. melanogaster, i.e., two strains of D. simulans, was considered.

In D. melanogaster, geographic populations are known to exhibit very different levels of polymorphism and allele frequencies at the Amy locus (Hickey, 1979; Singh et al., 1982). We have extended these geographical analyses to more numerous populations of that species and also to the seven other species included in the $D$. melanogaster taxonomic subgroup (see Lemeunier et al, 1986, for a review). We have found that $D$. melanogaster apparently harbors a generalised duplication with 12 different amylase variants identified. The other species are less polymorphic or monomorphic but, in four of them, a genetic analysis has demonstrated a duplication. It is thus suggested that among those species the duplication was an ancestral event whose evolutionary implications are discussed. 


\section{MATERIALS AND METHODS}

\section{Drosophila populations or strains}

Twenty seven natural populations of $D$. melanogaster and 18 of $D$. simulans were investigated (table 1). Electrophoresis was done either on wild caught individuals or on isofemale lines grown in the laboratory on corn meal medium. Two flies were scored for each isofemale line, after one or a few generations of laboratory culture. For the other species, one to three populations were used (table 1) and, for two of them, i.e., D. erecta and $D$. orena, only laboratory cultures were available. Moreover, for $D$. orena, the only existing strain has been founded by a single female.

Table 1 Electrophoretic polymorphism of amylase in the 8 species of the $D$. melanogaster subgroup: number of populations, individuals sampled and number of amylase isozy. mes identified (* For these species, only laboratory strains were available)

\begin{tabular}{llcc}
\hline Species & $\begin{array}{l}\text { Popula- } \\
\text { tions }\end{array}$ & $\begin{array}{l}\text { Indi- } \\
\text { viduals }\end{array}$ & $\begin{array}{l}\text { Iso- } \\
\text { amylases }\end{array}$ \\
\hline D. melanogaster & 27 & 3051 & 12 \\
D. simulans & 18 & 834 & 3 \\
D. mauritiana & 1 & 52 & 2 \\
D. sechellia & 1 & 76 & 3 \\
D. yakuba & 3 & 118 & 3 \\
D. teissieri & 1 & 40 & 2 \\
D. erecta* & 2 & 40 & 1 \\
D. orena* & 1 & 20 & 1 \\
Total & & 4231 & 17 \\
\hline
\end{tabular}

\section{Electrophoresis}

This was done on extracts of adult flies, using a 5 per cent polyacrylamide gel, with a $0.1 \mathrm{M}$ Trisborate buffer, $p \mathrm{H} 8.9$. After running (about 4 hours), gels were incubated for half an hour in $0.1 \mathrm{M}$ Tris- $\mathrm{HCl}(p \mathrm{H} \mathrm{7}$ ) containing 0.8 per cent of soluble starch. Gels were then washed and stained with a potassium iodide solution so that amylase activity appeared as white spots over a dark blue background.

\section{Genetic analysis}

Different techniques were used to investigate the occurrence of a duplication. For species which produce viable hybrids with $D$. melanogaster, crosses were done with an Amy null mutant of that species, in order to find haplotypes exhibiting two bands indicating that two genes are on a single chromosome. In other cases, the occurrence of individuals expressing three major amylase isozymes was investigated. Finally, when only two isoamylases were found, the duplication could be demonstrated by appropriate crosses and Mendelian analysis of the progeny. More details will be given in the results section.

\section{RESULTS}

\section{Electrophoretic variants among the 8 species}

Altogether, more than 4000 flies were studied, most of them belonging to the two cosmopolitan species, $D$. melanogaster and D. simulans, and the data are summarized in table 1.

As was previously known, D. melanogaster appears to be a very polymorphic species since 12 amylase isozymes were detected using only one electrophoretic technique. Among the 7 other species, 5 were found to be polymorphic, but with only 2 or 3 variants, and the final two were monomorphic. However, for these latter species, only laboratory strains were available so that it would be premature to draw any conclusion about their genetic variability. Considering the complete set of 8 species, several of them share isoamylases with very similar or identical mobilities so that, altogether, only 17 different amylase isozymes were identified. Their relative mobilities and designations are shown in fig. 1 and gel photographs are shown in fig. 2.

In $D$. melanogaster, six different mobility classes $(1,2,3,4,5,6)$ may be considered as the classical ones, having been observed by various investigators (Doane, 1969; Hickey, 1979; Singh et al., 1982; Yamazaki et al., 1984) and, of these, $A m y^{3}$ is generally the most frequent. In the literature, the rare $A m y^{7}$ allele was found by Singh et al., (1982) in an African population (Cotonou) and some other rare alleles, close to $A m y^{\prime}$ but presumably different from those shown in Figure 1, were described from America by Langley et al. (1974) and from Japan by Yamazaki et al. (1984). The five other polymorphic species (i.e., D. simulans, D. mauritiana, D. sechellia, D. yakuba and $D$. teissieri) exhibit altogether only five different isoamylases, $A m y^{4.4}$ (not found in $D$. melanogaster) being shared by all of them and being the most common in four species. $A m y^{3 \cdot 4}$ is also found in three of these species and in $D$. melanogaster, but only in Afrotropical populations. 


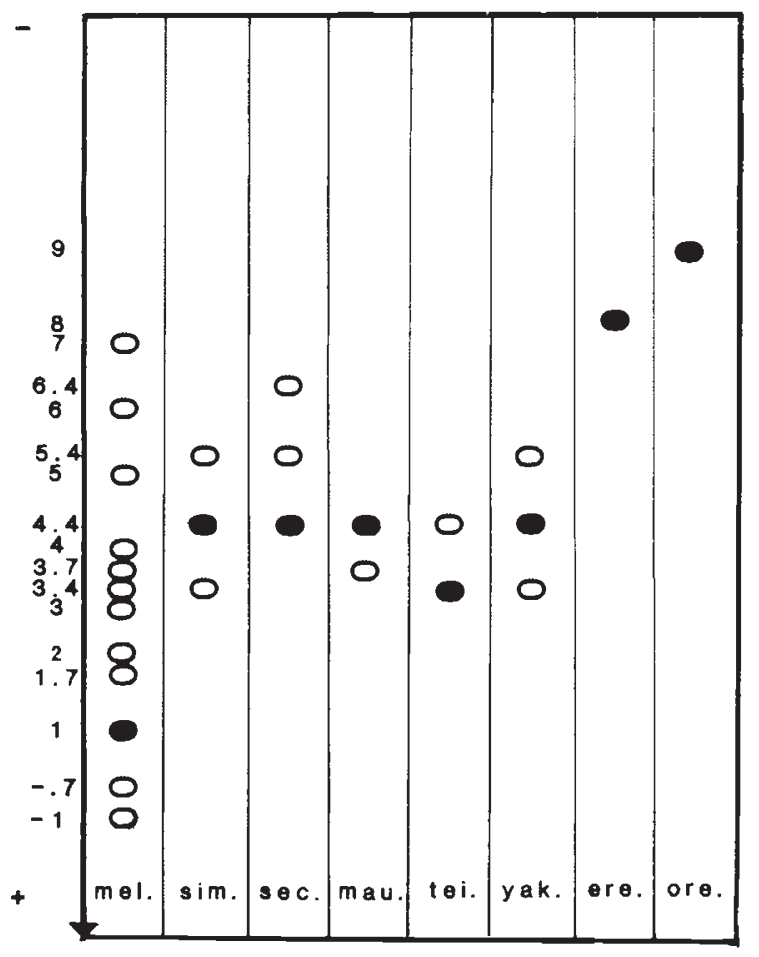

Figure 1 Diagrammatic representation of the occurrence and relative positions of the 17 different Amylase isozymes found among the 8 species of the D. melanogaster subgroup (mel: D. melanogaster; sim: D. simulans; sec: D. sechellia; mau: D. mauritiana; tei: D. teissieri; yak: D. yakuba; ere: $D$. erecta; ore: $D$. orena) (Under the experimental conditions used and after fixation of the gels, the distance between origin and allele 1 was $42 \mathrm{~mm}$ ). The usual nomenclature was used for the classical alleles. Those coding for proteins with intermediate mobility are numbered with decimals according to their relative distance between the classical alleles. For each species, the most common, or unique allele, is shown in black.

\section{The duplication in D. simulans}

The occurrence of a duplication was demonstrated in two ways. The first method involved the study of interspecific hybrids with an Amy null strain of D. melanogaster (Haj-Ahmad and Hickey, 1982). Males of a $D$. simulans line segregating for isoamylases $4 \cdot 4,5 \cdot 4$ were crossed with $D$. melanogaster, Amy null females. The hybrid progeny expressed only the paternal, D. simulans haplotype. The results (table 2) suggest that the $D$. simulans line segregated for two different chromosomes, one carrying $A m y^{4.4}$ and the other $A m y^{4.4}, A m y^{5.4}$.

The second method was to produce experimentally flies with a three-banded electrophoretic pattern. In D. simulans, $A m y^{3 \cdot 4}$ has been found in only one population from Antananarivo
(Madagascar). By inbreeding, a homozygous line $A m y^{3 \cdot 4}$ was obtained. This line was crossed with heterozygous individuals $A m y^{4 \cdot 4,5 \cdot 4}$ (table 2) and about half the progeny expressed three isozymes.

The observation of double-banded haplotypes and triple-banded heterozygotes provide strong evidence for a gene duplication, i.e., haplotypes expressing both $A m y^{4 \cdot 4}$ and $A m y^{5 \cdot 4}$.

\section{The duplication in D. mauritiana}

Two amylases variants were found in this species and only the hybridization with $D$. melanogaster Amy null strain was studied. D. mauritiana males carrying variants 3.7 and 4.4 were crossed with D. melanogaster Amy null females (table 2) and the offspring shows the association between $A m y^{3 \cdot 7}$ and $A m y^{4 \cdot 4}$.

\section{The duplication in D. yakuba}

This species cannot be hybridized to any of the others. However, it was possible to produce threebanded individuals by appropriate crosses. Many single pairs were isolated from a Kenyan population where the three amylase variants occurred and one of them was selected. The results given in table 2 are consistent with the following genetic interpretation of the parental cross:

$$
\begin{aligned}
& \text { q } A m y^{3 \cdot 4,4 \cdot 4} / A m y^{4 \cdot 4,4 \cdot 4} \\
& \times \delta^{7} A m y^{4 \cdot 4,5 \cdot 4} / A m y^{4 \cdot 4,4 \cdot 4}
\end{aligned}
$$

\section{The duplication in D. teissieri}

As in the case of D. yakuba, D. teissieri cannot be hybridized and only two electrophoretic variants, $A m y^{3 \cdot 4}$ and $A m y^{4 \cdot 4}$, were observed. However, a statistical analysis of some crosses again suggests the occurrence of a duplication. Among many random pairs, one in which both parents were doublebanded $A m y^{3 \cdot 4,4 \cdot 4}$ was chosen. In the F1, 53 individuals were electrophoresed and all exhibited identical phenotypes of $3 \cdot 4,4 \cdot 4$. From a mass F1 culture, $58 \mathrm{~F} 2$ individuals produced a phenotype of $3 \cdot 4,4 \cdot 4$, and 4 a phenotype of $3 \cdot 4$. Also, by an appropriate selection of $\mathrm{F} 2$ pairs, it was possible to obtain a line producing 100 per cent of the phenotype $3 \cdot 4,4 \cdot 4$ over successive generations.

This last result, i.e., a fixed double-banded pattern, could be due to a system of balanced recessive lethals closely linked to the $A m y$ locus, but such a system would imply the death of at least $50 \mathrm{per}$ cent of the zygotes. This interpretation was excluded by a viability study in this strain showing 

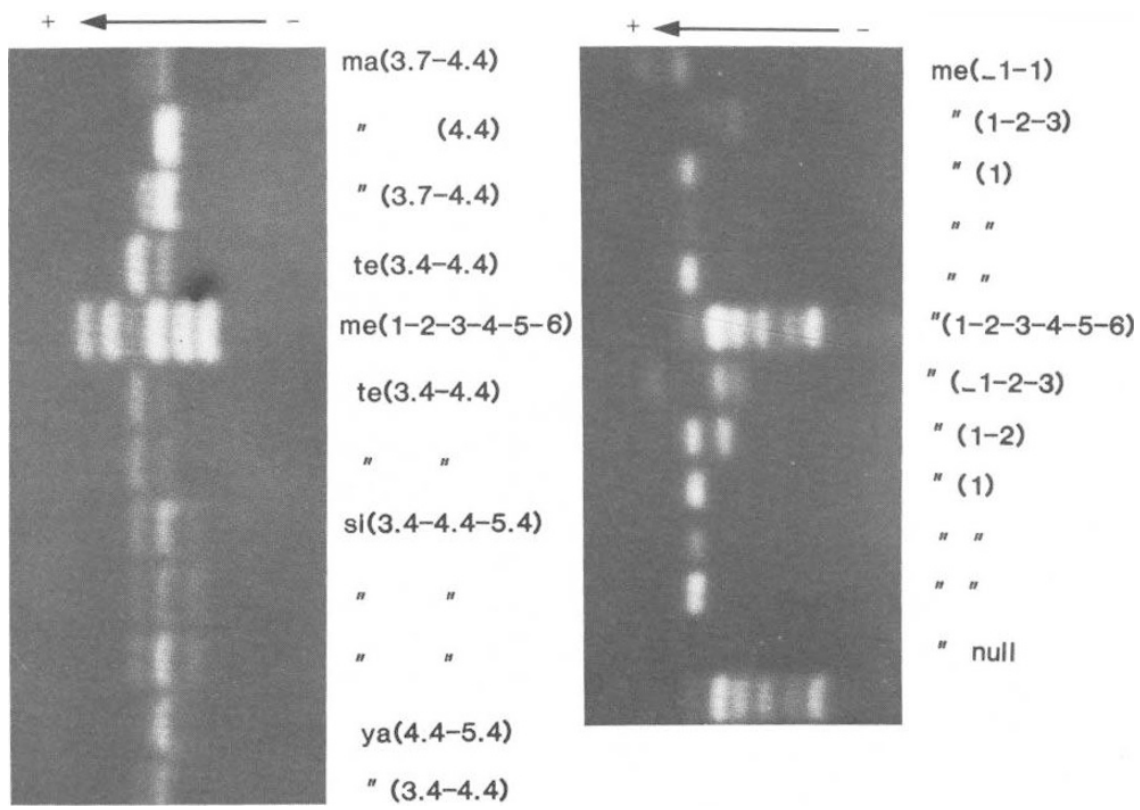

Figure 2 Amylase allozymes of several species within the $D$. melanogaster subgroup. The duplication is phenotypically expressed by three isoamylases with different electrophoretic mobilities. The melanogaster reference sample is a mixture of several individuals used as reference for electrophoretic mobility for the six classical alleles ( $A m y^{1}$ is the faster, $A m y^{6}$ the slower). ma: $D$. mauritiana: te: $D$. teissieri; me: $D$. melanogaster; si: $D$. simulans: ya: D. yakuba.

that on the average, about 80 per cent of eggs laid produced adults. On the other hand, the reappearance of about 6 per cent of $A m y^{3 \cdot 4}$ adults in the F2 is not consistent with the balanced lethals hypothesis. The most likely interpretation of the observed data is the presence of a duplication with the parental pair having the following genotypes:

$$
\begin{aligned}
& \text { q } A m y^{3 \cdot 4,4 \cdot 4} / A m y^{3 \cdot 4,4 \cdot 4} \\
& \times \delta^{\circ} A m y^{3 \cdot 4,4 \cdot 4} / A m y^{3 \cdot 4,3 \cdot 4}
\end{aligned}
$$

This cross would produce 100 per cent doublebanded phenotypes in the $F 1$ and a frequency of
6.25 per cent of $3 \cdot 4$ phenotypes in the F2.

\section{The case of $D$. sechellia}

Males from a mass culture segregating for alleles 4.4 and 5.4 were individually crossed with $D$. melanogaster Amy null females (table 2). The lack of double-banded haplotypes in the progeny suggests the absence of a functional duplication in $D$. sechellia although the hypothesis of duplicated homohaplotypes, i.e., $A m y^{4 \cdot 4,4 \cdot 4}$ and $A m y^{5 \cdot 4,5 \cdot 4}$, cannot be excluded.

Table 2 Inheritance of amylase variants in species of the Drosophila melanogaster subgroup. Crosses are presented in the same order as in text (D.m.: D. melanogaster; D.s.: D. simulans; D. mau: $D$.

\begin{tabular}{|c|c|c|}
\hline \multicolumn{2}{|l|}{ Parental phenotypes } & \multirow{2}{*}{ 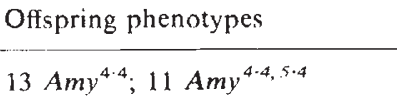 } \\
\hline @ D.m. Amy null & $\times \delta$ D.s. $A m y^{4 \cdot 4,5.4}$ & \\
\hline$q$ D.s. $A m y^{3 \cdot 4}$ & $\times \delta$ D.s. $A m y^{4 \cdot 4,5 \cdot 4}$ & $23 A m y^{3 \cdot 4 \cdot 4 \cdot 4} ; 25 A m y^{3 \cdot 4,4 \cdot 4,5 \cdot 4}$ \\
\hline I D.m. Amy null & $\times ð$ D. mau. Amy $y^{3 \cdot 7,4 \cdot 4}$ & $17 A m y^{4 \cdot 4} ; 14 A m y^{3 \cdot 7,4 \cdot 4}$ \\
\hline qD. yak. Amy $y^{3 \cdot 4,4 \cdot 4}$ & $\times \delta$ D. yak. $A m y^{4 \cdot 4,5 \cdot 4}$ & $\begin{array}{l}11 A m y^{3 \cdot 4,4 \cdot 4} ; 10 A m y^{3 \cdot 4,4 \cdot 4}, 5 \cdot 4 \\
13 A m y^{4 \cdot 4,5 \cdot 4} ; 14 A m y^{4 \cdot 4}\end{array}$ \\
\hline P. tei. $A m y^{3 \cdot 4,4 \cdot 4}$ & $\times \delta$ D. tei. $A m y^{3 \cdot 4,4 \cdot 4}$ & $\begin{array}{l}53 \mathrm{Amy}^{3 \cdot 4,4 \cdot 4}(\mathrm{~F} 1) \\
58 \mathrm{Amy}^{3 \cdot 4,4 \cdot 4} ; 4 \mathrm{Am} y^{3 \cdot 4}(\mathrm{~F} 2)\end{array}$ \\
\hline 9 D.m. Amy null & $\times 7$ 1). se. $A m y^{44,54}$ & $20 A m y^{4 \cdot 4}: 24 A m y^{5 \cdot 4}$ \\
\hline
\end{tabular}
mauritiana: D.yak.: D. yakuba; D. tei: D. teissieri; D.se.: D. sechellia) 


\section{DISCUSSION AND CONCLUSION}

We have presented evidence that the duplication at the Amy locus, which is well documented in $D$. melanogaster, also exists in four other related species. The lack of genetic evidence does not demonstrate the absence of the duplication in $D$. sechellia and also in the two presently monomorphic species, $D$. erecta and $D$. orena.

Dealing with a gene duplication raises two questions: $(a)$ is the duplication general on all chromosomes, even those which exhibit a single allele? (b) what is the location of the duplicated loci? In $D$. melanogaster, we have fairly good evidence to assume that the duplication is a general event also occurring in the homohaplotypes (Bahn, 1967; Hickey, 1979; Yamazaki et al., 1984; Daïnou, 1985; Levy et al., 1985). Recently, molecular studies provided evidence for the existence of the Amy duplication in D. melanogaster whether of not electrophoretic variation for amylase is expressed (Gemmill et al., 1986). In the same species, genetic studies indicated tha the two genes are very closely linked $(0.008 \mathrm{cM}, \mathrm{Bahn}, 1967)$ and located on the right arm on the second chromosome (Doane, 1967, 1969a, b). Considering that the $D$. simulans and D. mauritiana chromosome $2 \mathrm{R}$ does not differ from that of $D$. melanogaster (the species only differ by a single fixed inversion on chromosome 3R: see Lemeunier et al. (1986) for a review), it seems reasonable to assume that in the three species, the $A m y$ genes are carried by the same chromosome and have the same location. We may also suggest that in $D$. simulans and $D$. mauritiana the two genes are similarly closely linked, although a verification of this hypothesis by molecular analysis would be needed. In $D$. yakuba and $D$. teissieri, it is far more difficult to suggest any hypothesis about the localization of the Amy genes since these species differ from $D$. melanogaster by numerous fixed inversions. The generality of the duplication in all individuals within a species is still a more difficult problem.

Fig. 1 contrasts the great genetic diversity observed in $D$. melanogaster at the Amy locus with a much lower polymorphism in all other related species. We may argue that our sampling in $D$. melanogaster was much greater than in the other species (see table 1). However, if we consider the 11 Afrotropical populations of $D$. melanogaster, we usually find 6 to 9 different amylase variants in a sample of less than 100 flies (Daïnou, 1985) while a similar sample size of any other species will show only 2 or 3 isoamylases. Up to now, a likely interpretation of this contrast was the dupli- cation itself, supposed to exist only in $D$. melanogaster: if two loci are independently submitted to spontaneous mutagenesis, a doubling of the number of segregating alleles is expected. Results, presented here, show that such an explanation does not hold since the duplication also exists in less polymorphic species.

A possible interpretation is given by historical arguments. The Afrotropical region, especially West Africa, represents the ancestral home range of D. melanogaster where, although basically domestic, it displays the 'less domestic' ecological features known for the species all over the world. Accordingly, these populations could be expected to have undergone a long evolution that consequently generated a higher polymorphism.

With respect to its geographic populations, $D$. melanogaster is markedly more differentiated than D. simulans for chromosomal, quantitative, physiological, allozymic and DNA variation (Hyytia et al., 1985; Baba-Aissa and Solignac, 1984). At present the most likely hypothesis to explain the lower genetic variation within and between populations in $D$. simulans is to consider its reduced variability as a result of a more recent worldwide expansion. An alternative or additional interpretation to the higher variability of $D$. melanogaster would be to consider that even closely related species, may use different 'genomic strategies'. A chemical analysis of cuticular hydrocarbons in the 8 species of the $D$. melanogaster subgroup (Jallon and David, 1987) has shown tilat five species were very similar $(D$. simulans, $D$. mauritiana, $D$. yakuba, $D$. teissieri and $D$. orena) while $D$. melanogaster was much different. These observations suggest that after the cladogenesis, $D$. melanogaster 'evolved more rapidly' than its sibling species. Of course, this hypothesis would need further investigations.

Even if, during their evolutionary history, the related species used different genomic strategies and possibly different rates of evolution, it is interesting to consider their phylogeny. A classical interpretation (Lemeunier et al., 1986) mainly based on chromosomal, morphological and hybridization studies, divides the subgroup into two complexes (melanogaster and yakuba) each of four species, as shown in fig. 3A. The amylase data suggest, however, another classification, shown in fig. 3B. The first cladogenesis would separate the two species $D$. erecta and $D$. orena (both have very slow migrating amylase variants) from the six others. These six species are clustered together mainly because they partly share the same isoamylases $\left(A m y^{3 \cdot 4}\right.$ and $\left.A m y^{4 \cdot 4}\right)$ (fig. 1) and also 


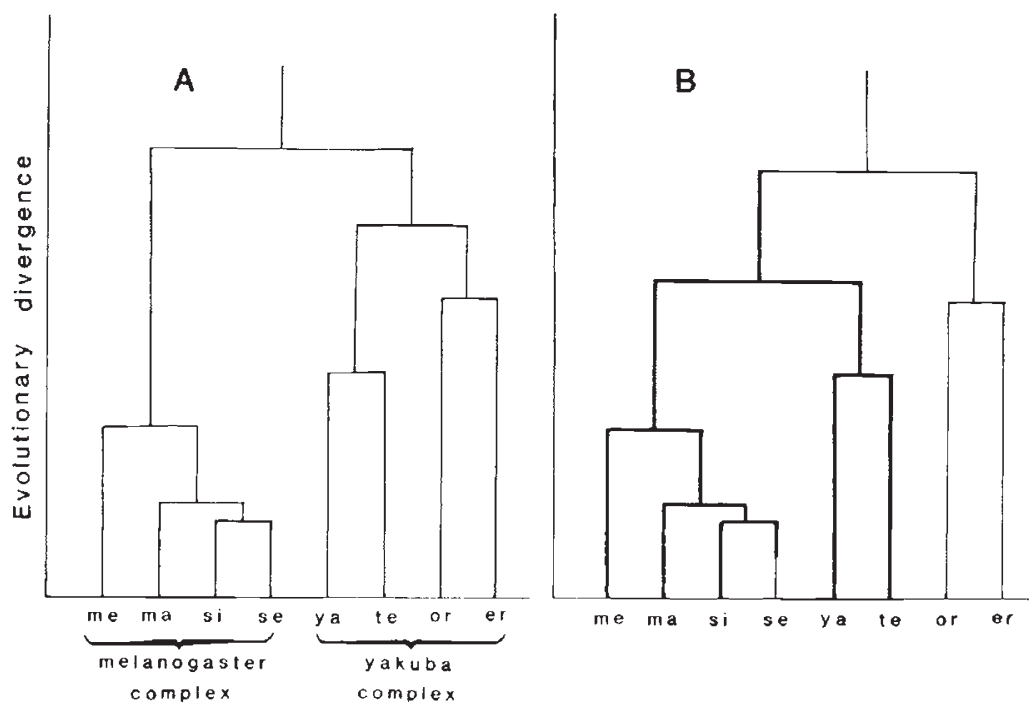

Figure 3 Phylogenetic cladograms for the 8 species of the $D$. melanogaster subgroup (me: D. melanogaster; ma: D. mauritiana: si: $D$. simulans; se: $D$. sechellia; ya: $D$. yakuba; te: D. teissieri; or: D. orena; er: D. erecta). A. Classical phylogeny based upon polytene chromosomes, dividing the subgroup into two complexes of four species. B. Alternative proposed phylogeny based upon the distribution of Amy alleles and consistent with allozymes, $2 \mathrm{D}$ electrophoresis and mitochondrial DNA data in placing $D$. erecta, $D$. orena apart (in heavy line, the duplication of the Amylase locus).

because they share the Amy locus duplication. Interestingly a similar cladogram is given by enzymes (Eisses et al., 1979), 2D electrophoresis (Ohnishi et al., 1983) and mitochondrial DNA data (Solignac et al., 1986); it is thus likely to correspond to the true phylogeny.

A question of evolutionary significance concerns the origin of the duplication. The distribution of the Amy duplication among related species suggestive of a single duplication event may be indicative of how old it is. The Amy locus is duplicated in five out of eight species of the melanogaster subgroup; it is thus reasonable to consider that the duplication is ancestral rather than the result of independent genetic events. Within the melanogaster complex the only exception is $D$. sechellia for which a secondary loss may be argued. It would be of great interest to confirm the apparent lack of Amy duplication in D. erecta and D. orena. If so, this would mean that the duplication has its earliest possible origin after the first cladogenesis within the melanogaster subgroup according to the fig. 3B phylogeny. Alternatively one might assume that the ancestral melanogaster lineage was polymorphic for the duplication; the lineage splitting off to give the erecta-orena pair lost the duplication while the lineage leading to the melanogaster- yakuba complex fixed the duplicated genes. On the contrary if, after further investigations of natural populations, $D$. erecta and $D$. orena appear to have the Amy duplication, its origin would be put off in a more ancient past, even before the radiation of the melanogaster subgroup. The recent finding of an Amy duplication in D. subobscura (Cariou et al., in preparation) i.e., in another taxonomic group in the Sophophora subgenus suggests such a possibility. Also, a male specific enzyme under independent genetic control and suggestive of a duplication has been identified in D. hydei (Doane et al., 1975). Further studies in other species of Drosophila are thus needed. Finally we might note that the occurrence of multiple amylase loci is also found in various mammals such as house mouse (Sick et al., 1964), bank vole (Nielsen, 1968), rabbit (Malacinski and Rutter, 1969) and Man (Karn et al., 1975).

\section{REFERENCES}

ABI: K. 1958. Genetical and biochemical studies in amylase in Drosophila melanogaster. Jap. J. Genet., 33, 138-145.

BAHN, E. 1967. Crossing over in the chromosomal region deter mining amylase isozymes in Drosophila melanngaster. Hereditas, $58,1-12$. 
BABA-AISSA, F. AND SOLIGNAC, M. 1984. La plupart des populations actuelles de Drosophila simulans ont probablement pour ancêtre une femelle unique dans un passé recent. $C$. R. Acad. Sci. Paris, 299, 289-292.

DAÏNOU, O. 1985. Polymorphisme et rôle physiologique de l'amylase chez Drosophila melanogaster et espèces affines. Thèse de 3ème cycle, Univ. Paris VII, 178 pp.

DOANE, w. W. 1965. Genetic control of amylase activity in Drosophila melanogaster. Proc. XII Int. Congr. Ent., London 1964, 233-234.

DOANE, w. W. 1967. Cytogenetic and biochemical studies of amylases in Drosophila melanogaster. Amer. Zool., 7, 780.

DOANE, W. W. 1969 a. Amylase variants in Drosophila melanogaster: linkage studies and characterization of enzyme extracts. J. Exp. Zool, 171, 321-342.

DOANE, W. W. 1969b. Drosophila amylases and problems in cellular differentiation. In RNA in Development, edited by E. W. Hanly, University of Utah Press, Salt Lake City, Utah, 73-109.

DOANE, W. W., ABRAHAM, i., KOLAR, M. M., MARTENSON, R. E. AND DEIBLER, G. E. 1975. Purified Drosophila-amylase isozymes: genetical, biochemical and molecular characterization. (ed. Markert, C. L.) in Isozymes IV. Genetics and Evolution. 585-607.

EISSES, K. T., DIJK, H. AND VAN DELDEN, W. 1979. Genetic differentiation within melanogaster species group of the genus Drosophila (Sophophora). Evolution, 33, 1063-1068.

GEMMILL, R. M., SCHWARTZ, P. E. AND DOANE, W. W. 1986. Structural organization of the Amy locus in seven strains of Drosophila melanogaster. Nucl. Acids Res., 14, 53375352.

HAJ-AHMAD, Y. AND HICKEY, D. A. 1982. A molecular explanation of frequency-dependent selection in Drosophila. Nature, 299, 350-352.

HICKEY, D. A. 1979. The geographical pattern of an enzyme polymorphism in D. melanogaster. Genetica, 51, 1-4.

HYYTIA, P., CAPY, P., DAVID, J. AND SINGH, R. S. 1985. Enzymatic and quantitative variation in European and African populations of Drosophila simulans. Heredity, 54, 209-217.

JALLON, J. M. AND DAVID, J. 1987 Variations in cuticular hydrocarbons among the eight species of the Drosophila melanogaster subgroup. Evolution 41, 294-302.
KARN, R. C., ROSENBLUM, B. B. AND MERRITT, A. D. 1975. Genetic and post-transcriptional mechanisms determining human amylase isozyme heterogeneity. Markert, C. L. (ed.) In Isozymes. IV. Genetics and Evolution, Academic Press, N.Y., pp. $745-761$.

KIKKAWA, H. 1960. Further studies on the genetic control of amylase in Drosophila melanogaster. Jap. J. Genet., 35, 382-387.

KIKKAWA, H. 1964. An electrophoretic study on amylase in Drosophila melanogaster. Jap. J. Genet., 39, 401-411.

LANGLEY, C. H., TOBARI, Y. N. AND KOJIMA, K. 1974. Linkage disequilibrium in natural populations of Drosophila melanogaster. Genetics, 78, 921-936.

LEMEUNIER, F., TSACAS, L., DAVID, J. AND ASHBURNER, M. 1986. The melanogaster species group. Ashburner, M., Thompson, J. R. Jr. and Carson, H. L. (eds) In The Genetics and Biology of Drosophila, Academic Press, London and New York, Vol. 3, 147-256.

LEVY, J. N., GEMMILl, R. M., AND DOANE, W. W. 1985. Molecular cloning of $\alpha$-amylase genes from Drosophila melanogaster. II. Clone organization and verification. Genetics, 110, 313-324.

MALACINSKY, G. M. AND RUTTER, W. J. 1969, Multiple molecular forms of $\alpha$-amylase from the rabbit. Bio chemistry, 8, 4382-4390.

NIELSEN, J. T. 1968. Genetic studies of the amylase isoenzymes of the bank vole, Clethrionomys glareola. Hereditas, 61, 400-412.

OHNISHI, S., KAWANISHI, M. AND WATANABE, T. K. 1983. Biochemical phylogenies of Drosophila: protein differences detected by two-dimensional electrophoresis. Genetica, 61, 55-63.

SICK, K. AND NIELSEN, J. T. 1964. Genetics of amylase isoenzymes in the mouse. Hereditas, 51, 291-296.

SINGH, R. S., HICKEY, I). A. AND DAVID, J. 1982. Genetic differentiation between geographically distant populations of Drosophila melanogaster. Genetics, 101, 235-256.

SOLIGNAC, M., MONNEROT, M. AND MOUNOLOU, J. C. 1986. Mitochondrial DNA evolution in the melanogaster species subgroup of Drosophila. J. Mol. Evol., 23, 31-40.

YAMAZAKI, T., MATSUO, Y., INOUE, Y. AND MATSUO, Y. 1984. Genetic analysis of natural populations of Drosophila melanogaster in Japan. I. Protein polymorphism, lethal gene, sterility gene, inversion polymorphism and disequilibrium. Jap. J. Genet, 59, 33-49. 